\title{
Der Kasus nach den lateinischen Präpositionen contra und per in der deutschen Sprache: Eine Korpusuntersuchung
}

\author{
Sophie Tschulik
}

The article examines the use of contra and per, two prepositions that originated from Latin and made their way into the German language. Using different corpora it analyses how these prepositions are currently applied and which case they require. These authentic language resources provide an insight into a topic which is neglected by traditional dictionaries and grammars. The article also covers the relevance of these findings for teaching German as a foreign language.

preposition - corpus - case - per - contra

Der Artikel thematisiert die Verwendung der zwei ursprünglich lateinischen Präpositionen contra und per in der deutschen Sprache. Unter Zuhilfenahme von Sprachkorpora wird der Frage nachgegangen, wie diese im Deutschen verwendet werden und welchen Kasus sie regieren. Diese authentischen Sprachquellen ermöglichen einen Einblick in ein von traditionellen Wörterbüchern und Grammatiken vernachlässigtes Thema. Berücksichtigt wird auch die Relevanz dieser Erkenntnisse für den DaF/DaZ-Unterricht.

Präposition - Sprachkorpus - Kasus - per - contra

\section{Einleitung}

Sowohl für Menschen, die mit Deutsch als Muttersprache aufgewachsen sind, als auch für jene, die sie erst erlernen, können sich Schwierigkeiten bei der korrekten Verwendung von Präpositionen, die die deutsche Sprache aus dem Lateinischen übernommen hat, ergeben. Meistens bieten Wörterbücher oder verschiedene Grammatiken bei Fragen beziehungsweise Problemen zu diesem Thema nur wenig Unterstützung, da sie sich vorrangig auf die „traditionellen deutschen“ Präpositionen sowie ihre Verwendung konzentrieren und zu den aus der lateinischen Sprache übernommenen häufig nur unzureichende oder einander widersprechende Informationen präsentieren. Dieser Beitrag will genau bei den Schwierigkeiten unterstützen, die sich beim Gebrauch ergeben können, und anhand von Korpusdaten die Problematik zweier ursprünglich lateinischer Präpositionen, die im Deutschen auftreten, beleuchten. Er befasst sich daher damit, wie diese in der gegenwärtigen deutschen Sprache eingesetzt werden und mit welchen Fällen sie kombiniert werden können. Ausgewählt wurden contra und per.

In alphabetischer Reihenfolge wird auf die zwei ausgewählten Wörter contra und per eingegangen. Zu Beginn werden der lateinische Gebrauch der entsprechenden Präposition dargelegt und einige Beispiele aus Texten Ciceros oder Caesars zur 
Veranschaulichung angeführt. Diese Stellen stammen aus der Library of Latin Texts. ${ }^{1}$ Dann werden die Erkenntnisse aus Wörterbüchern und Grammatiken vorgestellt, von denen sich eine explizit an $\mathrm{DaF} / \mathrm{DaZ}$-LernerInnen richtet. Anschließend werden das Korpus InterCorp und das Korpus Cosmas II beziehungsweise DeReKo des Instituts für Deutsche Sprache herangezogen, um Belegstellen aus authentischen Texten zu bekommen. Diese sollen Aufschluss darüber geben, wie in der aktuellen deutschen Sprache mit den ausgewählten Präpositionen umgegangen wird und welchen Kasus sie jeweils regieren.

\section{Präpositionen}

Präpositionen werden im Deutschen gemäß ihrer Funktion, eine Verbindung zwischen Wörtern herzustellen, auch als Verhältniswörter bezeichnet. (Grenzmer 1995: 271) In der Grammatik von Ludger Hoffmann findet sich folgende Definition: „Spezifizierungen des Gesagten werden im Deutschen oft durch relationierende Ausdrücke realisiert, durch Präpositionen, die Ausdrücke zu räumlicher oder zeitlicher bzw. einer anderen Spezifizierung ins Verhältnis setzen." (Hoffmann 2014: 372)

Jede Präposition kann einen beziehungsweise mehrere Kasus oder auch eine Infinitivgruppe regieren, ist aber selbst unveränderlich und kann nicht flektiert werden. (Hoffmann 2014: 372) Präpositionen sind also Partikeln. Sie lassen sich aber nicht nur anhand des Kasus, den sie verlangen, einteilen. Man kann auch zwischen freiem und gebundenem Gebrauch unterscheiden. Bei ersterem „entfaltet die Präposition ihre eigentliche Bedeutung und kann durch andere Präpositionen ersetzt werden." (Dudenredaktion und Wöllstein 2016: 614) Dies ist meistens bei adverbialen Ergänzungen sowie Angaben der Fall. (Dudenredaktion und Wöllstein 2016: 614) „Bei gebundenem Gebrauch verliert die Präposition ihre eigentliche Bedeutung und kann nicht mehr ausgetauscht werden." (Dudenredaktion und Wöllstein 2016: 614) Diese Regel gilt in idiomatisierten Wendungen bei Präpositionalobjekten, Funktionsverbgefügen und komplexen Adverbialen. (Dudenredaktion und Wöllstein 2016: 614)

Aus syntaktischer Sicht können Präpositionen allein noch nicht als Satzglied bezeichnet werden, sondern benötigen immer eine Ergänzung, bei der es sich meistens um eine Nominalphrase handelt. Andere Möglichkeiten wären eine Adjektivphrase oder eine Adverbphrase. (Dudenredaktion und Wöllstein 2016: 614) „Die Verbindung einer Präposition mit ihrer Ergänzung nennt man Präpositionalphrase, -gefüge oder -gruppe.“ (Dudenredaktion und Wöllstein 2016: 614)

Wie der Name Präposition bereits impliziert, stehen diese tatsächlich in den meisten Fällen vor ihrem Bezugswort, also jenem Wort, dessen Kasus sie regieren. Präpositionen

1 Die Library of Latin Texts ist eine Online-Datenbank lateinischer Texte, die ein spezifisches Suchen nach bestimmten Autoren, Wörtern oder Fällen ermöglicht. 
können allerdings auch in Postposition, also nachgestellt, oder in Zirkumposition auftreten. Bei letzterer Form wird das Bezugswort eingerahmt. (Dudenredaktion und Wöllstein 2016: 612) Präpositionen gelten als geschlossene Klasse. Man kann davon ausgehen, dass in der deutschen Sprache über 120 verschiedene Präpositionen verwendet werden. Regelmäßig in Sätzen treten aber nur ungefähr 20 Präpositionen, angeführt von in, mit, auf und von auf (Káňa 2014: 201).

\section{Die Präposition contra}

\subsection{Lateinische Verwendung}

Die lateinische Präposition contra bedeutet gegen(über) und verlangt im Lateinischen den Akkusativ. Sie wird sowohl örtlich zur Bezeichnung des Gegenüberliegenden gebraucht als auch im übertragenen Sinn ähnlich wie das deutsche Wort gegen. Diese zweite Verwendungsmöglichkeit ist laut Burkard und Schauer auch die häufigere. (Burkard und Schauer 2012: 256-257)

(1) auxilia ex Britannia, quae contra eas regiones posita est, arcessunt. ${ }^{1}$

(2) contra hostem ire iubet ${ }^{2}$ (LLT) $)^{3}$

\subsection{Angaben in Wörterbüchern}

Im Österreichischen Wörterbuch wird man zum Eintrag kontra verwiesen (Fussy und Steiner 2012: 151), der jedoch keine Informationen zum verlangten Kasus liefert, da bei dem angeführten Beispiel zwei Eigennamen verwendet werden. Stattdessen findet man die substantivierte Form des Wortes sowie die Phrase jemandem Kontra geben. (Fussy und Steiner 2012: 405) Der Duden führt das Wort ebenfalls unter kontra an, weist jedoch auch auf die Möglichkeit hin, die lateinische Variante zu verwenden. Abgesehen von einer Erklärung zur Bedeutung des Wortes finden sich aber keine Hinweise oder Regeln zum Gebrauch von kontra. Mit Beispielen belegt wird lediglich die substantivierte Verwendung (Dudenredaktion 2017: 655).

1 Sie holten die Hilfstruppen aus Britannien, das diesen Gebieten gegenüber liegt.

2 Er befahl gegen den Feind zu ziehen.

3 Um die Lesbarkeit des Beitrags zu erleichtern, wurde im Text darauf verzichtet, die verwendeten Corpora vollständig zu zitieren. Stattdessen wurde mit Abkürzungen gearbeitet. Die vollständige Internetadresse befindet sich im Literaturverzeichnis. 


\subsection{Angaben in Grammatiken}

Die Grammatik von Hoffmann (Hoffmann 2014: 384) liefert ebenso wie jene von Grenzmer (Grenzmer 1995: 271-292) und die Duden-Grammatik (Dudenredaktion und Wöllstein 2016: 405) keine Informationen zu contra.

\subsection{Korpusrecherche}

Das Korpus InterCorp findet 37 Treffer für contra, wobei nach Abzug der lateinischen Phrasen oder des nominalen Gebrauchs noch neun Fälle übrigbleiben, in denen dieses Wort auch als Präposition im Einsatz ist. Der Versuch mithilfe des Tagsystems nur an Belegstellen zu gelangen, in denen dem Wort ein Artikel beziehungsweise Adjektiv folgt, ist aufgrund mangelnder Ergebnisse fehlgeschlagen.

(3) Chef der konservativen Neuen Demokratie contra Alexis Tsipras, Chef des radikalen

(4) Baumarkt contra OBI

(5) Cornforth in seinem Buch Wissenschaft contra Idealismus unterlaufen ist. (InterCorp)

Bei all diesen Textausschnitten wird contra im Sinn von gegen gebraucht, auch wenn man manchmal einen Artikel ergänzen müsste, um bei einem Austausch der beiden Wörter weiterhin einen grammatikalisch korrekten deutschen Satz zu erhalten. Auffällig ist, dass contra fast immer verwendet wird, um zwei Dinge oppositionell gegenüberzustellen. Da dieser Gegensatz schlagwortartig und häufig bei Titeln und Überschriften gebraucht wird, fehlt der Einsatz von Artikeln oder Adjektiven, was es wiederum schwierig macht, den Kasus festzustellen. Aus pragmatischer Sicht bietet dies allerdings den Vorteil einer geringen Fehlerquelle.

Ebenfalls im InterCorp gibt es 44 Treffer für kontra, wobei nur in sechs Fällen eine Verwendung als Präposition vorliegt.

(6) beim ersten Mal: Zauber des Neuen kontra fehlende Eingespieltheit

(7) mehr Regulierung. Harmonisierung kontra gegenseitige Anerkennung (InterCorp)

Auch hier ist ein Tausch mit dem deutschen Wort gegen möglich. Da bei zwei Textstellen Adjektive zur genaueren Beschreibung eingesetzt werden, kann man Rückschlüsse auf den Kasus ziehen, den kontra beziehungsweise contra regiert. Es könnte sich bei diesen entweder um Wörter im Nominativ oder im Akkusativ handeln. Berücksichtigt man die Erkenntnisse des Instituts für Deutsche Sprache, regieren Präpositionen nie einen Nominativ, wodurch dieser Kasus ausscheidet. (Präpositionalphrasen IDS 2018) Allerdings wird in beiden Fällen die Präposition 
zur schlagwortartigen Gegenüberstellung verwendet, wodurch die Notwendigkeit eines vollständigen, grammatikalisch korrekten Satzes nicht gegeben ist.

Eine Suchabfrage im Korpus Cosmas II beziehungsweise DeReKo unter der Verwendung des Archivs TAGGED T, das morphosyntaktisch annotiert ist und ein einfacheres Recherchieren ermöglicht, findet 889 Textbelege, in denen das Wort contra vorkommt. Allerdings sind nicht alle Ergebnisse relevant, da nicht immer eine Verwendung als Präposition vorliegt. Dies beweist auch eine Kookkurrenzanalyse, die zeigt, dass contra vor allem in Kombination mit lateinischen Wörtern in Texten vertreten ist.

\begin{tabular}{llllll}
\hline$\#$ & LLR & kumul. & Häufig & Kookkurrenzen & syntagmatische Muster \\
\hline 1 & 231 & 9 & 9 & Templarios & $100 \%$ contra Templarios \\
2 & 205 & 17 & 8 & gentiles & $100 \%$ contra gentiles \\
3 & 188 & 27 & 10 & omnes & $100 \%$ contra omnes \\
4 & 180 & 35 & 8 & proprium factum & $100 \%$ contra factum proprium \\
5 & 69 & 41 & 6 & Transrapid & $100 \%$ contra Transrapid AcT \\
\hline
\end{tabular}

Abb. 1: Auswahl der Kookkurrenzen zur Abfrage contra (Cosmas II)

Weil im Archiv TAGGED T eine spezifische Suche nach den einzelnen Kasus nicht möglich ist und bei den meisten der 889 Belegstellen das Nomen direkt auf die räposition folgt, wurde speziell nach Treffern gesucht, die einen Artikel oder ein Adjektiv beinhalten. Dies erleichtert nämlich die eindeutige Identifikation des Falls. Sucht man also nach einer Verbindung von contra und einem Adjektiv, erhält man 50 Belegstellen. Dies ist natürlich ein großer Unterschied zu jenen 889, die man bekommt, wenn man lediglich nach der Präposition sucht. Dennoch ermöglichen es auch diese Treffer, einen Einblick in den authentischen Gebrauch zu erhalten und daraus Rückschlüsse auf eine generelle Verwendung zu ziehen.

(8) von Kultur zu: Moderne Welt contra traditionelle Welt, die in einer

(9) Direkte Abrechnung contra schlechte Zahlungsmoral (Cosmas II)

Diese Stellen sind Belege für den Gebrauch von contra in meist unvollständigen Sätzen zur Kontrastierung zweier Gegensätze und stehen im Akkusativ.

Eine Suche nach der Kombination von contra und einem Artikel, liefert 22 Belegstellen.

(10) Da ist der Stellungsbezug pro und contra eine Person unumgänglich, und den

(11) gehörten Argumenten pro oder contra den Proporz. (Cosmas II) 
Auch hier regiert contra einen Akkusativ. Auffällig ist, dass die Präposition gerne mit der antonymischen Präposition pro kombiniert wird.

Eine Abfrage mit der Schreibweise kontra liefert 1.439 Ergebnisse, wobei auch hier wieder etliche Treffer aus der Betrachtung ausgeschlossen werden können. Wie auch schon aus den Ergebnissen des InterCorp beziehungsweise jenen aus der ersten Abfrage des Cosmas II ersichtlich wurde, dominiert die Verwendung von contra im Kontext von Schlagworten. Diese Erkenntnis wird durch die 1.439 Ergebnisse mit kontra sowie eine erneute Kookkurrenzanalyse bestätigt. Der eingedeutschten Schreibweise geschuldet fehlen die lateinischen Ausdrücke, wie sie noch in der ersten Abbildung (Abb. 1) vertreten sind.

\begin{tabular}{llllll}
\hline$\#$ & LLR & kumul. & Häufig & Kookkurrenzen & syntagmatische Muster \\
\hline 1 & 70 & 4 & 4 & Einkaufsrummel & $100 \%$ kontra Einkaufsrummel geschäftiges \\
2 & 43 & 8 & 4 & Gentechnologie & $100 \%$ kontra Gentechnologie \\
3 & 42 & 11 & 3 & Kohlekraft & $100 \%$ kontra Kohlekraft \\
4 & 39 & 19 & 8 & Merkel & $87 \%$ kontra Merkel \\
5 & 37 & 23 & 4 & Windkraft & $75 \%$ kontra Windkraft \\
\hline
\end{tabular}

Abb. 2: Auswahl der Kookkurrenzen zur Abfrage kontra (Cosmas II)

Eine Suchabfrage, die nur Belegstellen anzeigt, in denen auf kontra ein Adjektiv folgt, liefert 38 Treffer.

(12) Massenware für den Supermarkt kontra authentisches Produkt.

(13) Mindestlohn kontra schärferes Jugendstrafrecht

(14) Pröll zu Konflikt Kommunismus kontra freie Marktwirtschaft (Cosmas II)

Auch wenn das Wort in seiner eingedeutschten Schreibweise eingesetzt wird, herrscht die Verwendung in kurzen Aussagen, Titeln, Phrasen oder Schlagworten vor, bei denen zwei Gegensätze gegenübergestellt werden und die keine vollständigen Sätze sind.

(15) Die weit verbreitete Emotion kontra grüne Gentechnik wird in

(16) und Pommes versus Salat, Gyros kontra gedünsteten Fisch: Um

(17) Partei St. Gallen spricht über pro und kontra freie Schulwahl. (Cosmas II)

Tritt die Präposition aber in vollständigen Sätzen auf, regiert sie einen Akkusativ. Diese Verwendungsmöglichkeit ist seltener.

13 Belegstellen findet Cosmas II, wenn man nach einer Verbindung von kontra und einem Artikel sucht, wobei diese im Bezug auf den Gebrauch keine neuen Erkenntnisse liefern. 
(18) die wissenschaftlichen Erkenntnisse kontra Mobilfunkanalagen noch nicht so weit

(19) der Amerikaner als Wanderprediger kontra die Diktatur in Iran (Cosmas II)

Trotz der Konzentration auf Ergebnisse, in denen den Präpositionen nicht nur ein Nomen folgt, soll darauf hingewiesen werden, dass es sowohl für contra als auch für kontra Belegstellen gibt, in denen die Präposition anlog zum Gebrauch in der lateinischen Sprache verwendet wird. Das heißt, sie dient nicht zur Gegenüberstellung von zwei Gegensätzen. Hinweise auf den verwendeten Kasus kann in diesem Fall allerdings nur der gesamte Satz geben, der zeigt, dass ein Akkusativ folgt.

\subsection{Fazit}

Auch wenn Wörterbucher nur sehr kurz auf den präpositionalen Gebrauch von contra eingehen und die Grammatiken ihn überhaupt nicht behandeln, hat die Recherche in den beiden Korpora gezeigt, dass die Präposition - abgesehen von gerne eingesetzten und auch in den Belegstellen zahlreich vertretenen lateinischen Phrasen wie beispielsweise contra legem - relativ häufig in der deutschen Sprache auftritt. So führt das Institut für Deutsche Sprache bei einer nach Häufigkeit gereihten Wortformenliste contra an 16.500. Stelle und kontra an 39.678. Stelle. (DeReWo IDS 2018) Die beiden Kookkurrenzanalysen haben auch demonstriert, dass auf contra meistens direkt ein Nomen ohne Zusatz folgt. Die Dominanz der alleinstehenden Nomen macht wiederum die eindeutige Identifikation des Kasus schwierig. Das mag zwar für Untersuchungen ungünstig sein, bietet aber eine geringere Fehlerquelle im allgemeinen Sprachgebrauch. Der Gebrauch eines Adjektivs ist noch häufiger als der eines Artikels. Dieser scheint nur in einer Kombination von pro und contra regelmäßig aufzutreten.

Wie die Belegstellen gezeigt haben, dominiert die Verwendung des Wortes zur Gegenüberstellung zweier Gegensätze in einem elliptischen Satz. In diesem Fall wird contra ohne Artikel verwendet und mit einem Akkusativ kombiniert. Wird contra hingegen in der lateinischen Tradition gebraucht, folgt ebenfalls ein Akkusativ. Auch wenn die Präposition in einem vollständigen und grammatikalisch korrekten Satz zur Kontrastierung zweier Begriffe eingesetzt wird, regiert sie diesen Kasus. Bezüglich der Häufigkeit des Auftretens muss zwischen den beiden Schreibweisen unterschieden werden. Für contra liefert Cosmas II eine Frequenz von $0.871 \mathrm{pMW}^{1}$, für kontra $1.411 \mathrm{pMW}$.

1 pro eine Million Worte (Das bedeutet, dass contra durchschnittlich $0.871 \mathrm{mal}$ pro 1.000 .000 Worte auftritt.) 
Beim Vergleich mit der lateinischen Sprache fällt auf, dass der lokale Gebrauch im Deutschen nicht mehr vorhanden ist, sondern die Präposition nur noch im übertragenen Sinn verwendet wird. Die Schreibweise betreffend tritt die eingedeutschte Variante kontra öfter auf.

\section{Die Präposition per}

\subsection{Lateinische Verwendung}

Die primäre Bedeutung der Präposition per lautet durch. Sie steht im Lateinischen vor einem Akkusativ und kann auf sechs verschiedene Arten eingesetzt werden. Bei örtlicher Verwendung bezeichnet sie eine durchlaufene Strecke und bei temporaler eine Zeitspanne. Ebenso kann durch sie ein Mittel beziehungsweise Werkzeug sowie eine Person oder Sache, die etwas ermöglicht, ausgedrückt werden. Per kann aber auch eine modale Bedeutung haben oder bei Schwüren in derselben Weise eingesetzt werden wie das deutsche Wort bei. (Burkard und Schauer 2012: 260-262)

(20) et per fines [...] in Apuliam peruenit ${ }^{1}$

(21) etiam per eos dies proelium [...] equestre fecit ${ }^{2}$

(22) certior ab Acilio et Murco per litteras factus ${ }^{3}$

(23) per me licet ${ }^{4}$

(24) qui etiam per iocum deos inridens 5

(25) per deos inmortales! ${ }^{6}$ (LLT)

\subsection{Angaben in Wörterbüchern}

Der Eintrag zu per im Österreichischen Wörterbuch ist ziemlich umfangreich und beinhaltet auch die Angabe des von der Präposition regierten Kasus. Wie im Lateinischen steht per auch bei deutschen Wörtern mit dem Akkusativ. Da bei den angeführten Beispielen allerdings ein Adjektiv fehlt und nirgendwo ein Artikel verwendet wird, ist der Fall bei Formulierungen wie per Adresse schwer festzustellen. (Fussy und Steiner 2012: 528) Der Duden beinhaltet einen langen Eintrag zu per, in dem auch darauf hingewiesen wird, dass die Präposition entweder mit dem Dativ oder mit dem Akkusativ verwendet werden kann. Allerdings gibt es nur ein Beispiel, in dem die beiden Fälle einander gegenübergestellt werden. Da bei den restlichen

1 Und er kam durch die Länder nach Apulien.

2 In diesen Tagen schlug er eine Reiterschlacht.

3 Er wurde von Acilius und Murcus durch Briefe informiert.

4 Meinetwegen!

5 Er verspottete im Scherz die Götter.

6 Bei den unsterblichen Göttern! 
wie auch im Österreichischen Wörterbuch kein Artikel gebraucht wird, kann auch hier nur schwer auf den Fall geschlossen werden. (Dudenredaktion 2017: 845)

\subsection{Angaben in Grammatiken}

In der Grammatik von Hoffmann wird darauf hingewiesen, dass per ein Medium, einen Weg oder ein Mittel ausdrückt und mit einem artikellosen Nomen im Akkusativ zu verwenden ist. (Hoffmann 2014: 386) Bei Grenzmer wird die Präposition per nicht besprochen. (Grenzmer 1995: 271-291) In der Duden-Grammatik wird darauf hingewiesen, dass per meistens einen Dativ und in seltenen Fällen auch einen Akkusativ regiert. (Dudenredaktion und Wöllstein 2016: 622-623)

\subsection{Korpusrecherche}

Die einfache Suchabfrage im InterCorp zur Präposition per präsentiert 3.549 Ergebnisse. Eine erste Analyse der Belegstellen ergibt, dass es sich bei dem Wort, das der Präposition per in einem Großteil der Fälle folgt, um ein artikelloses Nomen handelt. Das ist zwar interessant, wenn man sich für den allgemeinen Gebrauch interessiert, macht es jedoch praktisch unmöglich, bei Kombinationen wie beispielsweise per Post auf den Kasus zu schließen. Um diesen herauszufinden, muss also ein anderer Weg gewählt werden. So werden nur Belegstellen berücksichtigt, die entweder einen Artikel oder ein Adjektiv nach der Präposition aufweisen, auch wenn dies nicht der statistisch am häufigsten Variante mit bloßem Nomen entspricht. Denn nur auf diese Weise können Rückschlüsse auf den generellen Gebrauch von per gezogen werden.

Eine Suchabfrage mithilfe von Tags, die nur Treffer anzeigt, bei denen auf per ein Adjektiv folgt, liefert 93 Belegstellen. Nach dem Ausschluss aller italienischen Textbeispiele, bleiben noch 77 Treffer übrig.

(26) benötigten Informationen per elektronische Post übermittelt werden.

(27) Ultraschallsignale auf und funkt sie per bildgebendes System zurück. (InterCorp)

Bei 28 dieser 77 Belegstellen steht die Präposition per vor einem Akkusativ. Allerdings fällt auf, dass der Großteil der Treffer inhaltlich komplett deckungsgleich ist. Sortiert man diese Treffer aus, erhält man vier Fälle, in denen die Präposition einen Akkusativ regiert.

Das Korpus liefert 45 Belegstellen, in denen per ein Artikel und ein Nomen im Dativ folgen:

des Ein-Parteien-Systems nicht per allgemeinem Wahlrecht gewählt 
(29) Kommission benötigten Informationen per elektronischer Post übermittelt werden

(30) der exekutiven Funktionen und der per öffentlichem Gesetz (InterCorp)

Wenn man nur Belegstellen sucht, in denen per einem Artikel folgt, liefert InterCorp keine Treffer.

Eine Recherche im DeReKo liefert 149.320 Belegstellen, von denen ungefähr $3 \%$ wegen eines fehlenden Gebrauchs von per als Präposition ausgeschlossen werden können. Eine erste Durchsicht der Ergebnisse liefert ähnliche Erkenntnisse wie das Korpus InterCorp. Auch hier folgt der Präposition in den meisten Fällen direkt ein Nomen. Diesen Befund bestätigt auch eine Kookkurrenzanalyse.

\begin{tabular}{llllll}
\hline$\#$ & LLR & kumul. & Häufig & Kookkurrenzen & syntagmatische Muster \\
\hline 1 & 248352 & 22277 & 22277 & E-Mail & $99 \%$ per E-Mail an \\
2 & 89172 & 32490 & 10213 & Kopf & $99 \%$ per Kopf \\
3 & 69559 & 38129 & 5639 & Mail & $98 \%$ per Mail an \\
4 & 44433 & 42493 & 4364 & Fax & $94 \%$ per Fax unterlan \\
5 & 31877 & 46381 & 3888 & Post & $98 \%$ per Post an \\
\hline
\end{tabular}

Abb. 3: Auswahl der Kookkurrenzen zur Abfrage per (Cosmas II)

Erst an der 39. Stelle findet sich in der Tabelle mit per internationalem Haftbefehl ein Treffer, der nicht nur aus Präposition und Nomen besteht. Auch wenn es nicht der tatsächlichen Häufigkeit des Gebrauchs von per entspricht, konzentriert sich die Analyse auch bei der Recherche im Korpus DeReKo auf Belegstellen, die einen Artikel oder ein Adjektiv enthalten.

Eine Suchabfrage im Cosmas II mithilfe von Tags, die nur Treffer anzeigt, bei denen auf per ein Adjektiv folgt, liefert 8.261 Belegstellen. Um über eine repräsentative Stichprobe zu verfügen, wurden die ersten 1.000 Treffer gesichtet.

Nach dem Entfernen aller Treffer, die nur ein Datum ohne zusätzliches Adjektiv enthalten, bestätigt sich die aus dem InterCorp gewonnene Erkenntnis, dass per mit dem Akkusativ stehen kann.

(31) aufzuräumen, wird zunächst per rechte Maustaste auf eine freie Stelle der (32) die das Departement Couchepin per kommenden 1. Juli in Kraft gesetzt hat (33) 40 Prozent der per internationalen Haftbefehl gesuchten (Cosmas II)

Die zweite, häufiger anzutreffende Möglichkeit ist die Kombination von per mit dem Dativ. Statistisch gesehen steht in den untersuchten Belegstellen aus dem Korpus zu 72\% ein Dativ und zu 28\% ein Akkusativ. Diese Zahlen wurden aus den untersuchten 1.000 Treffern gewonnen. 
einer Normmeldeliste beziehungsweise per elektronischer Meldung bis spätestens

(35) Betroffene diese innerhalb einer Woche per eingeschriebenem Brief kündigen.

(36) besiegelte schon in der 49. Spielminute per erneutem Elfmeter den 3:1-Endstand.

(37) der Hausherren vergab Tibor Palla per abgefälschtem Freistoß. (Cosmas II)

Wie die folgenden Beispiele aber zeigen, ist die Wahl des Kasus nicht wirklich an bestimmte Regeln gebunden, da dieselben Formulierungen sowohl mit einem Akkusativ als auch mit einem Dativ nebeneinander existieren.

(38) 40 Prozent der per internationalen Haftbefehl gesuchten

(39) Der Mann ist untergetaucht, wird per internationalem Haftbefehl gesucht.

(40) nicht anerkannte Positionen diesem per eingeschriebenen Brief rügen.

(41) binnen einer Woche vom Vertrag per eingeschriebenem Brief zurücktreten (Cosmas II)

Eine Suchabfrage, die nur Belegstellen anzeigt, in denen auf per ein Artikel folgt, liefert lediglich fünf Treffer, wobei auch nicht immer eine präpositionale Verwendung vorliegt. Bei diesen wurde ein Akkusativ gebraucht.

\subsection{Fazit}

Eine Durchsicht der Wörterbücher und verschiedenen Grammatiken hat bereits gezeigt, dass unterschiedliche Meinungen zum Gebrauch der Präposition per in der deutschen Sprache existieren. So gibt es Werke, die entweder keine Angaben machen oder den Gebrauch eines Akkusativs vorschreiben und sogar solche, die meinen, dass sowohl der Akkusativ als auch der Dativ nach per stehen könne. Eine Recherche in den beiden Korpora hat gezeigt, dass anhand des tatsächlichen Sprachgebrauchs der letzten Gruppe recht zu geben ist.

Mit Abstand am häufigsten ist jene Variante, in der per direkt ein Nomen folgt. Dies haben sowohl die Ergebnisse aus dem InterCorp als auch di Kookkurrenzanalysen im Cosmas II ergeben. Diese primäre Verwendung von per macht es zwar einerseits schwierig, den Kasus zu analysieren, hat aber auch andererseits einen Vorteil, da aufgrund der häufig deckungsgleichen Formen eines deutschen Wortes in den verschiedenen Fällen die Fehlerquelle relativ gering ist. Da kaum Belege gefunden wurden, in dem hinter der Präposition ein Artikel folgt, kann man davon ausgehen, dass diese Variante in der deutschen Sprache nicht gebräuchlich ist. Die Analyse der Fälle hat gezeigt, dass per immer entweder einen Akkusativ oder einen Dativ regiert. Mit ungefähr $72 \%$ überwiegt aber die Verwendung des letzteren Kasus eindeutig. Bezüglich der Häufigkeit des Auftretens der Präposition liefert Cosmas II eine Frequenz von 146.4 pMW.

Im Vergleich mit der lateinischen Sprache fällt auf, dass die lokale Bedeutung im Deutschen nicht mehr aufscheint, die temporale schon. Allerdings wird nun 
nicht mehr eine Zeitspanne, sondern ein Zeitpunkt ausgedrückt. Ebenfalls nicht mehr im Einsatz ist der modale Gebrauch und auch Personen werden kaum mit per kombiniert. Durchgesetzt hat sich die Verwendung der Präposition zur Bezeichnung eines Mittels oder eines Werkzeugs. Was die Wahl des Kasus angeht, folgt im klassischen Latein immer ein Akkusativ, in der deutschen Sprache kann man hingegen zwischen diesem und dem Dativ wählen.

\section{Conclusio}

Bei der Betrachtung der Einträge in den Wörterbüchern hat sich gezeigt, dass die Angaben entweder nicht ausreichen, um daraus Rückschlüsse auf ihre korrekte Verwendung in der deutschen Sprache zu ziehen, oder einander widersprechen. Zu einem ähnlichen Befund kommt man auch nach der Durchsicht der ausgewählten Grammatiken, die contra und per überwiegend nicht behandeln.

Die Recherche in den beiden Korpora InterCorp und Cosmas II ermöglicht es, einen Einblick in den authentischen Sprachgebrauch zu erhalten und daraus auch Rückschlüsse auf grammatikalische Regeln zu ziehen. Die Suchabfragen und Frequenzen zu den ausgewählten Präpositionen haben gezeigt, dass beide - wenn auch in unterschiedlichem Ausmaß - einen festen Platz in der deutschen Sprache gefunden haben. Besonders bei per konnten sehr viele Belegstellen nachgewiesen werden. Diese Präposition steht meist mit dem Dativ, manchmal auch mit einem Akkusativ, während contra stets einen Akkusativ regiert. Dies stellt eine klare Tendenz dar.

In einem Vergleich mit der lateinischen Sprache hat sich anhand der Korpora gezeigt, dass sich die Verwendung sowohl bezüglich des Kasus als auch bezüglich des konkreten Gebrauchs stark gewandelt hat. Im Gegensatz zur lateinischen Verwendung, bei der die Wahl des Kasus streng geregelt ist, ist der Einsatz im Deutschen weniger strikt definiert. Dabei ist allerdings anzumerken, dass die Präpositionen zwar mehrere Fälle regieren, der konkrete Gebrauch aber in der deutschen Sprache eingeschränkter ist und die verschiedenen Verwendungsmöglichkeiten weniger zahlreich sind.

Im Hinblick auf künftige Arbeiten zu ähnlichen Themen, wäre es interessant, sich einerseits mit weiteren lateinischen Präpositionen zu beschäftigen und andererseits mit Wörtern wie beispielsweise via, die erst in der deutschen Sprache eine präpositionale Verwendung erfahren haben. Auch eine Korpusrecherche, die sich spezifisch mit regionalen Unterschieden bezüglich des gewählten Kasus beschäftigt, wäre aufschlussreich. Auf dieses Thema konnte im vorliegenden Artikel nicht näher eingegangen werden.

Bezüglich des Einsatzes der gewonnenen Ergebnisse für das Unterrichten von Deutschlernenden lässt sich sagen, dass der Fokus des DaF/DaZ-Unterrichts natürlich auf den „klassischen“ deutschen Präpositionen liegt. Da sich die Verwendung 
der lateinischen Präpositionen einfach vermeiden lässt, müssen diese gerade in der Anfangsphase des Spracherwerbs nicht explizit thematisiert werden. Dennoch sind die behandelten Präpositionen Teil der Bildungssprache und sollten als solche ab dem Niveau B1 angesprochen werden. Ebenfalls können Deutschlernende in Büchern, Artikeln und sonstigen Texten auf diese stoßen und danach Fragen zu ihrem Gebrauch haben. Der vorliegende Artikel sieht sich daher als Hilfestellung und möchte bei der Beantwortung eben jener Fragen unterstützen.

\section{Literaturverzeichnis}

Burkard, Thorsten / Schauer, Markus: (52012): Lehrbuch der lateinischen Syntax und Semantik. Begründet von Hermann Menge. Darmstadt, Wissenschaftliche Buchgesellschaft.

Dudenredaktion (Hg.) (272017): Duden. Die deutsche Rechtschreibung. Berlin, Dudenverlag.

Dudenredaktion / Wöllstein, Angelika (Hg.) ('2016): Duden. Die Grammatik. Berlin, Dudenverlag.

Fussy, Herbert / Steiner, Ulrike (Hg.) ( $\left.{ }^{42} 2012\right)$ : Österreichisches Wörterbuch. Wien, Österreichischer Bundesverlag.

Grenzmer, Herbert (1995): Deutsche Grammatik. Frankfurt am Main [u.a.], Insel Verlag.

Hoffmann, Ludger (2014): Deutsche Grammatik. Grundlagen für Lehrerausbildung, Schule, Deutsch als Zweitsprache und Deutsch als Fremdsprache. Berlin, Erich Schmidt Verlag.

Hofmann, Johann Baptist / Rubenbauer, Hans (122014): Lateinische Grammatik. Neubearbeitet von R. Heine. Bamberg, C.C. Buchners Verlag [u.a.].

Káňa, Tomás (2014): Sprachkorpora in Unterricht und Forschung DaF/DaZ. Brno, Masarykova univerzita.

\section{Internetquellen}

Präpositionalphrasen. Einfache Strukturen. Mannheim, Institut für Deutsche Sprache. https:// grammis.ids-mannheim.de/systematische-grammatik/1422 (19.02.18).

DeReWo - Korpusbasierte Grund-/Wortformenlisten. Mannheim, Institut für Deutsche Sprache. http://wwwl.ids-mannheim.de/kl/projekte/methoden/derewo.html (25.02.18)

\section{Sprachkorpora}

Cosmas II (2017). Korpus Search, Management and Analysis System. Mannheim, Institut für Deutsche Sprache. www.ids-mannheim.de/DeReKo (10.02.18).

InterCorp (2016). Korpus InterCorp. Praha, Univerzita Karlova. http://www.korpus.cz/ intercorp/ (10.02.18).

LLT- Library of Latin texts [Series A] (2017). Library of Latin Texts. Brepols Publishers. http:// clt.brepolis.net.uaccess.univie.ac.at/llta/pages/QuickSearch.aspx (15.02.18).

Sophie Tschulik

Universität Wien

E-Mail: Sophie.tschulik@chello.at

Brünner Hefte zu Deutsch als Fremdsprache • Jahrgang 11・Nummer 2 • 2018 